\title{
Colitis ulcerosa crónica idiopática: epidemiología, características clínicas y factores asociados al tratamiento quirúrgico en un hospital de tercer nivel en México
}

\author{
Ulcerative colitis: epidemiology, clinical features and associated factors with surgical \\ treatment in a tertiary hospital in Mexico
}

\section{Jorge L. de León-Rendón ${ }^{1}$, Billy Jiménez-Bobadilla', Raquel Y. López-Pérez², Noé I. Gracida-Mancilla ${ }^{3}$, Lisbeth Alarcón-Bernés ${ }^{3}$ y Juan A. Villanueva Herrero ${ }^{1 *}$}

${ }^{1}$ Servicio de Coloproctología; ${ }^{2}$ Servicio de Gastroenterología; ${ }^{3}$ Servicio de Cirugía General. Hospital General de México Dr. Eduardo Liceaga, Ciudad de México, México

\begin{abstract}
Resumen
Antecedentes: Se tiene evidencia de un aumento en la incidencia de la colitis ulcerosa crónica idiopática (CUCl) en México, pero varía según el Estado e incluso entre hospitales de una misma región. Es importante conocer las características clínicas de los pacientes con CUCl, su curso clínico, necesidad de tratamiento quirúrgico y desenlaces. Objetivo: Describir las características demográficas y clínicas de los pacientes con CUCl, enfatizando en los factores asociados a la necesidad de tratamiento quirúrgico. Método: Estudio retrospectivo que incluyó 60 pacientes con diagnóstico de CUCl confirmado por histopatología. Se colectaron los datos demográficos, clínicos y bioquímicos de cada paciente. La gravedad se midió con la escala de Truelove y Witts, la escala endoscópica de Mayo y el índice histológico de Riley. Resultados: Se incluyeron 30 hombres y 30 mujeres. La media de edad fue de $40.6 \pm 13.3$ años. La extensión de la enfermedad fue proctitis en el $61.7 \%$. Diecinueve pacientes (31.8\%) presentaron manifestaciones extraintestinales. El 55\% tenían como tratamiento único ácido 5-aminosalicílico. Catorce pacientes $(23.3 \%)$ requirieron tratamiento quirúrgico, principalmente por falta de respuesta al tratamiento médico. Conclusiones: Las características clínicas de la $\mathrm{CUCl}$ son útiles para identificar a los pacientes que pudieran presentar una evolución tórpida de la enfermedad y requerir tratamiento quirúrgico.
\end{abstract}

PALABRAS CLAVE: Colitis ulcerosa crónica idiopática. Colon. Enfermedad inflamatoria intestinal. Epidemiología. México.

\begin{abstract}
Background: An increase incidence of ulcerative colitis (UC) has been reported in Mexico. It is important to know the clinical features of patients with UC, their clinical course, need for surgical treatment and outcomes. Objective: To describe the demographic and clinical features of patients with UC, emphasizing the factors associated with the need for surgical treatment. Method: A retrospective, cross-sectional, analytical study was made which included 60 patients with diagnosis of UC confirmed by histopathology. The demographic, clinical and biochemical data of each patient will be collected. The severity was measured based on the Truelove and Witts scale, endoscopic Mayo score and Riley histological index. Results: Of the patients included, the proportion was equal between men and women. The mean age of presentation was $40.6 \pm 13.3$ years. The extent
\end{abstract}

\author{
Correspondencia: \\ *Juan A. Villanueva Herrero \\ Dr. Balmis, 148 \\ Col. Doctores, Del. Cuauhtémoc \\ C.P. 06726, Ciudad de México, México \\ E-mail: javillanueva@doctor.com
}

Fecha de recepción: 13-09-2018

Fecha de aceptación: 15-12-2018

DOI: $10.24875 / C I R U .18000737$
Cir Cir. 2019;87:450-458

Contents available at PubMed www.cirugiaycirujanos.com 
of the disease at the time of diagnosis was proctitis in the majority of patients (61.7\%). Nineteen patients (31.8\%) presented extraintestinal manifestations. $55 \%$ of the patients had as sole treatment 5 -aminosalicylates. Fourteen patients $(23.3 \%)$ required surgical treatment mainly due to lack of response to medical treatment. Conclusions: The clinical features of UC are useful to identify patients who may present a torpid evolution of the disease and who may require surgical treatment.

KEY WORDS: Colon. Epidemiology. Inflammatory bowel disease. Mexico. Ulcerative colitis.

\section{Introducción}

La colitis ulcerosa crónica idiopática $(\mathrm{CUCl})$ es un subtipo de enfermedad inflamatoria intestinal caracterizada por la inflamación de la mucosa colónica que se extiende de manera proximal y continua a partir del recto ${ }^{1-3}$.

La incidencia de la enfermedad inflamatoria intestinal varía desde el punto de vista geográfico y es mayor en el norte y el oeste de Europa, así como en el norte de América; las zonas geográficas de menor incidencia son África, América del Sur, este de Europa y Asia, incluida China ${ }^{4}$. En las últimas décadas se ha encontrado un aumento en la incidencia de la enfermedad inflamatoria intestinal en los países del este de Europa y en las naciones asiáticas ${ }^{5}$. Lo anterior se atribuye a la occidentalización de los estilos de vida, los cambios en la dieta y los cambios ambientales debidos a la industrialización y la urbanización de las poblaciones $^{6}$.

Un estudio realizado en un hospital especializado de la Ciudad de México, donde se hizo una revisión de expedientes de 20 años, encontró un aumento estadísticamente significativo de $\mathrm{CUCl}$ en los últimos 10 años ${ }^{7}$. Existen pocos reportes acerca de la epidemiología de la $\mathrm{CUCl}$ en América Latina y específicamente en nuestro país.

Los síntomas y la gravedad de esta enfermedad dependerán de la extensión, el grado de inflamación y la actividad de las manifestaciones extraintestinales asociadas, aspectos que le confieren una heterogeneidad clínica significativa ${ }^{1,2}$.

A pesar de los avances recientes en la terapia médica para la $\mathrm{CUCl}$, entre un 15 y un $30 \%$ de los pacientes requerirán en algún momento tratamiento quirúrgico ${ }^{8,9}$.

En el presente estudio se pretende describir las características demográficas y clínicas de los pacientes con diagnóstico de $\mathrm{CUCl}$ atendidos en un hospital de tercer nivel en la Ciudad de México, enfatizando en los factores asociados a la necesidad de tratamiento quirúrgico.

\section{Método}

Se realizó un estudio retrospectivo, observacional, analítico, de pacientes con diagnóstico de $\mathrm{CUCl}$ atendidos en el servicio de coloproctología del Hospital General de México Dr. Eduardo Liceaga, en el periodo comprendido entre noviembre de 2016 y octubre de 2017. Se incluyeron pacientes con edad mayor de 18 años, de ambos sexos, mestizos mexicanos, con diagnóstico de CUCI confirmado por histología.

Se realizó la revisión de expedientes clínicos para obtener las siguientes variables: edad, sexo, edad al diagnóstico, extensión de la enfermedad, manifestaciones extraintestinales, curso clínico, tratamiento médico y necesidad de tratamiento quirúrgico, biometría hemática, química sanguínea, albúmina, colesterol, proteína $C$ reactiva y velocidad de sedimentación globular. El curso clínico de la enfermedad se definió de la siguiente manera: activo y luego inactivo (primer episodio seguido de una remisión de largo plazo por más de 5 años), intermitente (menos de dos recaídas por año) y actividad crónica (actividad persistente a pesar del tratamiento médico). La respuesta al tratamiento médico se categorizó de la siguiente manera: favorable, dependiente de esteroides (recaída al ajustar la prednisona a menos de 15mg/día o 3 meses después de suspender el esteroide); resistente al esteroide (actividad persistente con prednisona con al menos $0.5 \mathrm{mg} / \mathrm{kg} / \mathrm{día}$ ); y resistente a los inmunomoduladores (ausencia de ahorro de esteroides con una dosis de azatioprina de por lo menos $2 \mathrm{mg} / \mathrm{kg} / \mathrm{día}$ por más de 3 meses). La gravedad de la enfermedad fue medida mediante la escala de Truelove y Witts (clínica), la subescala endoscópica de Mayo y el índice histológico de Riley. Los pacientes se agruparon en aquellos con que cursaban con actividad leve, moderada o grave según los criterios establecidos por cada una de las escalas evaluadas.

Se revisaron 102 expedientes clínicos, de los cuales 42 fueron excluidos. Se excluyó a los pacientes con otro tipo de enfermedades inflamatorias del colon: enfermedad de Crohn, colitis indeterminada, colitis infecciosa o colitis microscópica. También se excluyó 
a los pacientes sin diagnóstico confirmado por histología de $\mathrm{CUCl}$ y a aquellos cuyo expediente clínico estuviera incompleto.

El estudio fue revisado y avalado por el comité de ética e investigación del hospital.

Se calcularon medidas de tendencia central y dispersión para las variables cuantitativas, y razón de momios con intervalos de confianza utilizando la prueba de ji al cuadrado. Las variables cuantitativas se analizaron mediante la prueba t de Student. Se consideró significativo un valor de $p \leq 0.05$. El análisis de los datos estadísticos se realizó con el paquete estadístico SPSS versión 17.

\section{Resultados}

Se incluyeron 60 pacientes con $\mathrm{CUCl}$ (30 hombres y 30 mujeres). La media de edad de presentación fue de $40.6 \pm 13.3$ años (rango: 19-72). El 61\% (37) de los pacientes presentaron la enfermedad antes de los 40 años. El tiempo de evolución de la enfermedad fue de $4.07 \pm 2.77$ años (rango: 1-14). Ocho pacientes (13.3\%) eran fumadores activos. La extensión de la enfermedad al momento del diagnóstico estaba limitada al recto en el $61.7 \%$ de los pacientes, el $30 \%$ presentaban pancolitis y el $8.3 \%$ tenían colitis izquierda. En el $45 \%$ de los pacientes se encontró un curso intermitente de la enfermedad, en el $36.7 \%$ un curso activo y posteriormente inactivo, y el $18.3 \%$ presentó actividad continua. En cuanto a la gravedad de la enfermedad valorada por la escala de Truelove y Witts, el $8.3 \%$ era inactiva, el $18.3 \%$ mostraba actividad leve, el $60 \%$ actividad moderada y el $13.3 \%$ actividad grave. La actividad endoscópica medida por la escala de Mayo fue inactiva en el $1.7 \%$, leve en el $31.7 \%$, moderada en el $31.7 \%$ y grave en el $35 \%$. La actividad histológica por el índice de Riley fue inactiva en el $6.7 \%$, leve en el $26.7 \%$, moderada en el $48.3 \%$ y grave en el $18.3 \%$. Diecinueve pacientes $(31.8 \%)$ presentaron manifestaciones extraintestinales de la enfermedad: artralgias $(26.7 \%)$, colangitis esclerosante primaria $(1.7 \%)$, espondilitis anquilosante $(1.7 \%)$ y pioderma gangrenoso $(1.7 \%)$. Treinta y tres pacientes $(55 \%)$ tenían como tratamiento único ácido 5-aminosalicílico (5-ASA); el resto de los pacientes, además del tratamiento con 5-ASA recibían esteroides (35\%), azatioprina (8.3\%) o terapia biológica (1.7\%). La evolución de los pacientes fue favorable con el tratamiento médico establecido (en su mayoría 5-ASA) en el $78.3 \%$ de los casos, y el $21.7 \%$ no tuvieron una evolución favorable; de estos pacientes, el $13.3 \%$ presentaron dependencia de
Tabla 1. Características clínicas y demográficas de los pacientes $(n=60)$ con colitis ulcerosa crónica idiopática

\begin{tabular}{lc}
\hline Edad media, años & $40.63 \pm 13.35$ \\
Mínima & 19 \\
Máxima & 72 \\
Sexo, $n$ (\%) & \\
Masculino & $30(50)$ \\
Femenino & $30(50)$ \\
Tabaquismo positivo, $n$ (\%) & $8(13.3)$ \\
Edad al diagnóstico <40 años & $37(61.6)$ \\
Años de evolución & \\
Mínimo & $4.07 \pm 2.77$ \\
Máximo & 01 \\
Extensión & 14 \\
Proctitis & \\
Colitis izquierda & $37(61.7)$ \\
Pancolitis & $05(08.3)$ \\
Actividad clínica, $n(\%)$ & $18(30.0)$ \\
Activo-inactivo & \\
Intermitente & \\
Actividad continua & $22(36.7)$ \\
Manifestaciones extraintestinales, $n(\%)$ & $27(45.0)$ \\
Sí & $11(18.3)$ \\
No & \\
Frecuencia manifestaciones extraintestinales, $n(\%)$ & \\
Artralgias/artritis & $16(26.7)$ \\
Colangitis esclerosante primaria & $01(01.7)$ \\
Espondilitis & $01(01.7)$ \\
Pioderma gangrenoso & $01(01.7)$
\end{tabular}

Actividad clínica, n (\%)

Escala de Truelove y Witts

Inactiva

Leve

Moderada

05 (08.30)

$11(18.30)$

$36(60.00)$

Grave

$08(13.30)$

Actividad endoscópica, n (\%)

Escala de Mayo

Inactiva

Leve

Moderada

Grave

$01(01.70)$

$19(31.70)$

$19(31.70)$

$21(35.00)$

Actividad histológica, n (\%)

Índice de Riley

Inactiva

Leve

Moderada

Grave

$04(06.70)$

$16(26.70)$

$29(48.30)$

$11(18.30)$

Tratamiento médico, n (\%)

5-ASA

$33(55)$

5-ASA+esteroides

5-ASA+tiopurinas $\quad 05(8.3)$

Biológico 01 (1.7)

Tratamiento quirúrgico, $\mathrm{n}$ (\%)

Sí

$14(23.3)$

$46(76.7)$ 
Tabla 1. Características clínicas y demográficas de los pacientes ( $n=60)$ con colitis ulcerosa crónica idiopática (Continuación)

Indicación de tratamiento quirúrgico, n (\%)

No respuesta a tratamiento médico

Sangrado masivo

Perforación

Displasia

$10(16.6)$

$02(3.3)$

$01(1.7)$

$01(1.7)$

Procedimiento quirúrgico, $\mathrm{n}(\%)$

Proctocolectomía+ileostomía

Proctocolectomía total+reservorio en J

Colectomía subtotal+colostomía colectomía

total+ileostomía

$6(10.0)$

$3(5.0)$

$3(5.0)$

$2(3.3)$

esteroides, el $6.7 \%$ resistencia a esteroides y el $1.7 \%$ resistencia a tiopurinas. Catorce pacientes (23.3\%) requirieron tratamiento quirúrgico, la mayoría de ellos por una respuesta no favorable al tratamiento médico establecido. Todos los pacientes que requirieron tratamiento quirúrgico no tenían la posibilidad económica de adquirir inmunomoduladores o biológicos (16.7\%). La segunda causa de cirugía fue el sangrado masivo, en el $3.3 \%$ de los casos. Estos últimos se presentaron con rectorragia, estado de choque y necesidad de transfusión de al menos dos paquetes globulares cada 24 horas, y sin respuesta a la terapia con esteroides intravenosos (evaluada mediante los criterios de Oxford). Por último, como motivos de cirugía se tuvo la perforación colónica secundaria a megacolon tóxico $(1.7 \%)$ y la presencia de adenocarcinoma de colon sigmoides poco diferenciado (1.7\%); este paciente fue diagnosticado por colonoscopia en una fase inactiva de la enfermedad. Los procedimientos quirúrgicos realizados fueron proctocolectomía total con ileostomía terminal $(10.0 \%)$, proctocolectomía total con reservorio en J $(5.0 \%)$, colectomía subtotal con ileostomía terminal $(5.0 \%)$ y colectomía total con ileostomía terminal (3.3\%). Durante el periodo de seguimiento, solo una paciente presentó estenosis del sitio de anastomosis del reservorio ileal. No se registró mortalidad asociada a los eventos quirúrgicos. Las características clínicas y demográficas de los pacientes se resumen en la tabla 1.

\section{Tratamiento quirúrgico}

El curso clínico de la enfermedad en este grupo de pacientes fue intermitente en el $42.9 \%$ y con actividad persistente en el $57.1 \%$ de los casos (odds ratio [OR]: 19.11; intervalo de confianza del 95\% [IC 95\%]: $3.9-92.60 ; p<0.0001)$. Ninguno de los pacientes operados presentó curso activo y después inactivo. La extensión de la enfermedad en el grupo de pacientes

operados se distribuyó de la siguiente manera: proctosigmoiditis (2 pacientes, 14.3\%), colitis izquierda (1 paciente, $7.1 \%$ ) y pancolitis (11 pacientes, $78.6 \%$ ), siendo la pancolitis la extensión más frecuente en estos pacientes $(p=0.000)$ y un factor de riesgo para requerir tratamiento quirúrgico (OR: 24.44; IC 95\%: 5.2-113.81; $p<0.0001$ ). Las causas por las cuales dos de los pacientes con actividad limitada (proctosigmoiditis) requirieron tratamiento quirúrgico fueron sangrado masivo y presencia de adenocarcinoma de colon sigmoides poco diferenciado.

Referente al tratamiento médico establecido al momento de requerir tratamiento quirúrgico, 10 pacientes (71.4\%) se encontraban en tratamiento con esteroides (OR: 13.71; IC 95\%: 2.70-69.53; p < 0.0001); solo uno (7.1\%) de los pacientes operados se encontraba con tratamiento con 5-ASA y recibió tratamiento quirúrgico por la presencia de adenocarcinoma de colon sigmoides poco diferenciado. Dos pacientes (14.2\%) se encontraban en tratamiento con inmunomoduladores y uno $(7.1 \%)$ con terapia biológica.

Se operaron más pacientes con actividad grave clasificada por la escala endoscópica de Mayo que por actividad grave medida por la escala de Truelove y Witts o por la clasificación histológica de Riley. De los pacientes operados, el $28.6 \%$ fueron catalogados con actividad grave por la escala de Truelove y Witts (OR: 4.22; IC 95\%: 0.89-19.74; $p=0.77$ ). Sin embargo, cuando se analiza el mismo grupo con la escala endoscópica de Mayo, este se elevó al 64.3\% (OR: 5.1; IC 95\%: 11.4-118.27; $p=0.01$ ), y según la actividad histológica (índice de Riley) al 57.1\% (OR: 19.11; IC 95\%: 3.94-92.60; $p<0.0001)$. Las características clínicas y demográficas de los pacientes con CUCI tratados quirúrgicamente se muestran en la tabla 2.

Al comparar las características clínicas y demográficas de los pacientes que requirieron tratamiento quirúrgico contra los que no lo requirieron, no encontramos diferencia significativa entre sexo, edad, índice de masa corporal, tabaquismo y la presencia de manifestaciones extraintestinales.

En cuanto al número de hospitalizaciones en los pacientes que requirieron cirugía respecto a los tratados medicamente, no encontramos diferencias entre ambos grupos (no operados $1.50 \pm 3.29$ hospitalizaciones vs. operados $3.21 \pm 1.12$ hospitalizaciones; $p=0.06$ ). Sin embargo, todos los pacientes operados requirieron por lo menos una hospitalización durante el curso de la enfermedad (OR: 1.8; IC 95\%: 1.41-2.39; $p<0.0001$ ). 
Tabla 2. Características clínicas y demográficas de los pacientes con colitis ulcerosa crónica idiopática que requirieron tratamiento quirúrgico

\begin{tabular}{|c|c|c|c|c|}
\hline & $\begin{array}{c}\text { Operados } \\
(n=14 ; 23.33 \%)\end{array}$ & $\begin{array}{c}\text { No operados } \\
(n=46 ; 76.67 \%)\end{array}$ & $p$ & OR (IC 95\%) \\
\hline \multicolumn{5}{|l|}{ Sexo } \\
\hline Masculino & 7 & 23 & \multirow[t]{2}{*}{0.61} & \\
\hline Femenino & 7 & 23 & & \\
\hline Edad, años & $39.07 \pm 11.52$ & $41.11 \pm 13.94$ & 0.62 & - \\
\hline Índice de masa corporal, kg/m2 & $24.97 \pm 5.21$ & $24.37 \pm 4.54$ & 0.68 & - \\
\hline \multicolumn{5}{|l|}{ Tabaquismo } \\
\hline Positivo & 1 & 7 & \multirow[t]{2}{*}{0.66} & \\
\hline Negativo & 13 & 39 & & \\
\hline \multicolumn{5}{|l|}{ Manifestaciones extraintestinales } \\
\hline Sí & 7 & 12 & \multirow[t]{2}{*}{0.89} & \\
\hline No & 7 & 34 & & \\
\hline \multicolumn{5}{|l|}{ Hospitalizaciones } \\
\hline No & 0 & 21 & \multirow[t]{2}{*}{0.001} & \multirow[t]{2}{*}{$1.8(1.41-2.39)$} \\
\hline$>1$ & 14 & 25 & & \\
\hline \multicolumn{5}{|l|}{ Extensión de la enfermedad } \\
\hline Proctitis & 2 & 35 & \multirow[t]{3}{*}{0.0001} & \multirow[t]{3}{*}{$24.44(5.2-113.81)$} \\
\hline Colitis izquierda & 1 & 5 & & \\
\hline Pancolitis & 11 & 6 & & \\
\hline \multicolumn{5}{|l|}{ Curso clínico } \\
\hline Activo y después inactivo & 0 & 22 & \multirow[t]{3}{*}{0.0001} & \multirow[t]{3}{*}{$19.11(3.9-92.60)$} \\
\hline Actividad intermitente & 6 & 21 & & \\
\hline Actividad persistente & 8 & 23 & & \\
\hline \multicolumn{5}{|l|}{ Tratamiento médico } \\
\hline 5-ASA & 1 & 32 & \multirow[t]{4}{*}{0.0001} & \multirow[t]{4}{*}{$13.71(2.70-69.53)$} \\
\hline Esteroides & 10 & 14 & & \\
\hline Inmunomoduladores & 2 & 0 & & \\
\hline Biológico & 1 & 0 & & \\
\hline \multicolumn{5}{|c|}{ Gravedad (escala de Truelove y Witts) } \\
\hline Inactiva & 0 & 5 & \multirow[t]{4}{*}{0.77} & \\
\hline Leve & 1 & 10 & & \\
\hline Moderada & 9 & 27 & & \\
\hline Grave & 4 & 4 & & \\
\hline \multicolumn{5}{|c|}{ Gravedad endoscópica (escala Mayo) } \\
\hline Inactiva & 0 & 1 & \multirow[t]{4}{*}{0.01} & \multirow[t]{4}{*}{$5.1(11.4-118.27)$} \\
\hline Leve & 1 & 18 & & \\
\hline Moderada & 4 & 15 & & \\
\hline Grave & 9 & 12 & & \\
\hline \multicolumn{5}{|c|}{ Gravedad histológica (índice de Riley) } \\
\hline Inactiva & 0 & 4 & \multirow[t]{4}{*}{0.0001} & $19.11(3.94-92.60)$ \\
\hline Leve & 1 & 15 & & \\
\hline Moderada & 5 & 24 & & \\
\hline Grave & 8 & 3 & & \\
\hline
\end{tabular}

\section{Discusión}

La CUCl es el subtipo de enfermedad inflamatoria intestinal con mayor incidencia en México ${ }^{7}$. Lo anterior se atribuye a la occidentalización del estilo de vida ${ }^{6}$, mismo que influye en los hábitos de alimentación (dieta rica en hidratos de carbono y grasas) ${ }^{10-13}$. Se describen otros factores de riesgo para el desarrollo de la enfermedad, tales como tabaquismo, estrés, efectos secundarios por la industrialización (contaminación del aire, agua, entre otros) y estrato socioeconómico alto; esto representa el impacto de los 
factores ambientales para el desarrollo de la enfermedad ${ }^{10-13}$.

Se han descrito diferentes alteraciones inmunogenéticas en la población mexicana que pudieran predisponer al desarrollo de CUCl14-16.

Nuestro estudio describe una población de pacientes con $\mathrm{CUCl}$ atendidos en el servicio de coloproctología de un hospital de tercer nivel de atención en la capital del país, que es centro de referencia nacional para patologías colorrectales.

En la población estudiada observamos una distribución similar entre ambos sexos, y más de la mitad de los pacientes fueron diagnosticados antes de los 40 años de edad; datos similares a los reportados en otros estudios realizados en nuestro país ${ }^{7,17}$. En las últimas décadas se ha observado un incremento en la incidencia de $\mathrm{CUCl}$ en diferentes grupos de edad, pero la mayoría de los pacientes con $\mathrm{CUCl}$ se encuentran en los 30-40 años al momento del diagnóstico ${ }^{18}$. Se ha observado que la edad promedio al momento del diagnóstico suele ser un poco mayor en los países asiáticos que en los países occidentales ${ }^{19}$.

Entre los factores estudiados, el tabaquismo se ha descrito como factor protector para desarrollar CUCI. Los estudios han descrito que el riesgo de los no fumadores para desarrollar $\mathrm{CUCl}$ es aproximadamente tres veces mayor que el de los fumadores ${ }^{20}$. Además, parece ser un factor protector contra la colectomía (OR: 0.57; IC 95\%: 0.38-0.85) y contra el desarrollo de cáncer colorrectal (OR: 0.5; IC 95\%: 0.2-0.92)21,22. En nuestro estudio hubo predominio de pacientes no fumadores, similar a lo reportado en otras series.

De acuerdo con la clasificación de Montreal, según la extensión, la $\mathrm{CUCl}$ puede clasificarse en tres subtipos diferentes: proctitis (cuando la inflamación está restringida a la mucosa del recto), colitis izquierda (cuando la inflamación se extiende más allá del recto y la flexura esplénica) y extensa (cuando la inflamación alcanza la mucosa proximal a la flexura espléni$\left(\mathrm{ca}^{23}\right.$. Estudios realizados en otros países, así como en México, reportan diferentes frecuencias en la extensión de la enfermedad ${ }^{24-27}$. En nuestro país se ha reportado pancolitis en más del $50 \%$ de los $\operatorname{casos}^{7,17}$. En el presente estudio encontramos con mayor frecuencia proctitis como extensión de la enfermedad, seguida de pancolitis y finalmente de colitis izquierda. Nuestros resultados son coincidentes con lo descrito en algunos países, como China $(7.3 \%)^{28}$, Irán $(18.1 \%)^{29}$ y Brasili27.

Le escala de Truelove y Witts es considerada simple y útil en la práctica clínica, y es aplicable a pacientes con enfermedad extensa, pero probablemente no refleja de manera adecuada la gravedad de la enfermedad en los pacientes con colitis limitada ${ }^{17}$. En nuestro estudio, más del $50 \%$ de los pacientes presentaron actividad moderada por la escala de Truelove y Witts.

La colonoscopia permite la evaluación directa de las lesiones de la mucosa intestinal en los pacientes con CUCl. La gravedad de las lesiones refleja en general la actividad clínica de la enfermedad y permite identificar a los pacientes que tienen una mayor probabilidad de desarrollar un peor comportamiento de la enfermedad con el tiempo ${ }^{30}$.

Existen algunos métodos que permiten establecer la actividad de la CUCl. Uno de ellos es el índice histológico de Riley, que muestra principalmente la densidad y la distribución de los neutrófilos en los defectos mucosos ${ }^{31}$.

En los pacientes con $\mathrm{CUCl}$ de nuestro estudio, la actividad endoscópica (medida con la escala de Mayo) se comportó como grave, mientras que la actividad histológica fue moderada en casi el $50 \%$ de los casos. Algunos estudios reportan una correlación entre la clasificación endoscópica y los hallazgos histológicos encontrados en la clasificación microscópica de Riley ${ }^{31,32}$. Sin embargo, en nuestro estudio, las escalas de gravedad clínica-bioquímica, endoscópica e histológica no presentan correlación entre sí.

La frecuencia de manifestaciones extraintestinales se ha reportado entre un 6 y un $40 \%$, dependiendo de las poblaciones estudiadas. En los países occidentales y en algunos estudios realizados en India y en nuestro país se ha reportado una frecuencia del 21 al $41 \%{ }^{7,30}$. En otros países se han reportado tasas menores, como China $(6.1 \%)$ y Corea $(24.1 \%)^{7,30}$. En nuestro estudio, 19 (31.8\%) pacientes presentaron manifestaciones extraintestinales de la enfermedad, siendo las reumatológicas las más frecuentes (26.7\%), de modo similar a lo reportado en todas las series.

La mayoría de los pacientes eran tratados con 5-ASA como tratamiento de primera línea, seguido de corticoides orales e inmunomoduladores, de modo similar a lo reportado en estudios realizados en China, India y en nuestro país $\mathbf{s}^{7,733-35}$. Solo el $1.7 \%$ de nuestros pacientes recibió terapia biológica (mismo que requirió tratamiento quirúrgico).

Se ha descrito que aproximadamente el $27 \%$ de los pacientes con CUCl hospitalizados de urgencia requerirán colectomía debido a una falta de respuesta al tratamiento médico o secundario al desarrollo de complicaciones propias de la $\mathrm{CUCl}$. Alrededor del 
69\% de los pacientes con CUCl grave responderán al uso de esteroides intravenosos. No obstante, en más del $56 \%$ de los pacientes que no responden a esta terapia se inicia tratamiento de rescate, de acuerdo con los criterios de Oxford, con infliximab o ciclosporina $^{36}$. La terapia con infliximab es una opción para los pacientes con CUCl grave refractaria a los esteroides intravenosos, como una alternativa para evitar la colectomía ${ }^{37}$.

Consideramos que un porcentaje de colectomías realizadas en nuestra institución se deben a una mala respuesta al tratamiento con esteroides intravenosos en los pacientes hospitalizados con $\mathrm{CUCl}$ grave, no siendo posible escalar la terapia médica a fármaco biológico por no contar con este recurso en nuestro hospital, o bien por no poder ser costeado por el paciente, requiriendo así tratamiento quirúrgico.

Una revisión sistemática publicada por Stawowczyk y Kawalec ${ }^{38}$ refiere que los ensayos clínicos revelan que los productos biológicos son eficaces en la terapia médica de la $\mathrm{CUCl}$, pero que también generan una pesada carga para los proveedores de servicios de salud. Los índices de costo-utilidad incrementales para los productos biológicos, en comparación con la atención estándar, varían significativamente entre los estudios, desde 36,309 hasta $\$ 456,979$. Se obtuvo el valor más bajo para infliximab y el más alto para el esquema de tratamiento que incluye infliximab $5 \mathrm{mg} /$ $\mathrm{kg}$ e infliximab $10 \mathrm{mg} / \mathrm{kg}$ más adalimumab ${ }^{38}$.

La prevalencia creciente y los altos costos de la farmacoterapia y de la cirugía en la CUCI constituyen un desafío considerable para los sistemas de salud y generan la necesidad de un manejo eficiente y rentable de esta enfermedad crónica a largo plazo. En este sentido, creemos que se requiere la eficacia en función de los costos de las diferentes estrategias de tratamiento para tomar decisiones de reembolso objetivas y verificables, que brinden a los pacientes los mejores regímenes de tratamiento disponibles. Considerando que la mayoría de los pacientes con $\mathrm{CUCl}$ en nuestro país se encuentran en una edad productiva, debe evaluarse el impacto de las nuevas intervenciones (terapia biológica) en la productividad del paciente y la correspondiente carga económica para la sociedad. Así mismo, hay que tomar en cuenta que el retraso en el tratamiento o en la ejecución de tratamientos con mayor morbilidad en estos pacientes podría repercutir en una importante pérdida de productividad, y por lo tanto, los costos indirectos podrían incluso exceder los costos directos de salud.
Por otro lado, la presencia de neoplasia solo se encontró en un paciente $(1.7 \%)$, similar a lo reportado en población iraní y estadounidense, pero se han descrito tasas de cáncer colorrectal más altas en población caucásica ${ }^{11,29}$.

La tasa de colectomía fue del $23.3 \%$ en nuestro estudio, similar a lo reportado en la literatura internacional y nacional (7.5-20\%), después de 5 años de evolución de la enfermedad 39,40 .

En nuestro estudio, en el subgrupo de pacientes que requirieron tratamiento quirúrgico no observamos diferencias en cuanto al sexo y la edad en comparación con aquellos que fueron manejados de forma conservadora. Existen estudios que refieren el sexo masculino y la edad mayor de 50 años como factores de riesgo para requerir tratamiento quirúrgico durante el curso de la enfermedad; así mismo, señalan que el sexo femenino representa un factor protector para la necesidad de tratamiento quirúrgico ${ }^{41}$. Sin embargo, algunos reportes no encuentran diferencias en cuanto al sexo como factor de riesgo para colectomía, y el riesgo por edad lo expresan variable entre los 35 y los 64 años $^{41-43}$.

Algunos otros factores, como la obesidad y la presencia de manifestaciones extraintestinales, se han relacionado con la necesidad de tratamiento médico más agresivo, en particular con la prescripción temprana de inmunosupresores o de terapia biológica para evitar los efectos adversos de la terapia con esteroides; así como un riesgo mayor de requerir tratamiento quirúrgico ${ }^{44,45}$. En nuestro estudio no encontramos asociación entre el índice de masa corporal o la presencia de manifestaciones extraintestinales con la necesidad de colectomía.

En nuestros pacientes, el tabaquismo no influyó en la necesidad de colectomía, hallazgo que concuerda con lo reportado en el estudio IBSEN y en otros, que no mostraron ningún efecto del tabaquismo sobre el riesgo de colectomía durante los primeros 10 años de diagnóstico de la $\mathrm{CUCl}^{46-48}$.

Se ha descrito que los pacientes con $\mathrm{CUCl}$ que necesitan ser hospitalizados durante el curso de la enfermedad tienen más riesgo de requerir colectomía ${ }^{41}$; en nuestro estudio, la totalidad de los pacientes que requirieron tratamiento quirúrgico tuvieron por lo menos una hospitalización.

La mayoría de nuestros pacientes operados presentaban pancolitis como extensión de la enfermedad, el curso clínico se comportaba como intermitente o persistente, y eran tratados con esteroides. Nuestros hallazgos coinciden con lo reportado en la literatura, en la que se describe que los pacientes con $\mathrm{CUCl}$ 
hospitalizados en cualquier momento tenían un mayor riesgo de colectomía ${ }^{41}$. Se ha encontrado una asociación entre el uso de corticosteroides y la colectomía. Los pacientes que tomaron corticosteroides (por vía oral o intravenosa) durante el curso de la enfermedad tuvieron un mayor riesgo de colectomía ${ }^{41}$. Finalmente, existen reportes sobre los cuatro patrones predefinidos de enfermedad, que muestran que más de la mitad de los pacientes con $\mathrm{CUCl}$ no colectomizados presentaron remisión o sintomatología leve después de un periodo de actividad inicial, mientras que el 37 y el $6 \%$ fueron reportados con actividad intermitente y persistente, respectivamente ${ }^{46}$.

En general se utilizan los parámetros clínicos, bioquímicos, endoscópicos e histológicos para medir la gravedad de la $\mathrm{CUCl}^{49}$. El diagnóstico de la $\mathrm{CUCl}$ grave se basa en el índice de Truelove y Witts, el cual es el más simple, mejor validado y ampliamente utilizado para la clasificación clínica de la $\mathrm{CUCl}^{37}$. Sin embargo, es bien sabido que la actividad inflamatoria histológica persiste a pesar de un adecuado control de los síntomas y de la ausencia de actividad endoscópica. Es importante recordar que la actividad clínica puede correlacionarse pobremente con la actividad histológica y endoscópica ${ }^{37,49}$.

De los pacientes operados en nuestro estudio, solo el $28.6 \%$ fueron catalogados como graves por la escala de Truelove y Witts. Sin embargo, cuando se analiza el mismo grupo con la escala endoscópica de Mayo, el porcentaje se elevó al $64.3 \%$, y según la actividad histológica (índice de Riley) al 57.1\%.

La endoscopia desempeña un papel muy importante en la evaluación de la $\mathrm{CUCl}$ en los brotes agudos; una rectosigmoidoscopia flexible es suficiente para evaluar la gravedad y obtener biopsias. La presencia de ulceraciones profundas, enfermedad extensa o friabilidad de la mucosa en un estudio endoscópico corresponde a una enfermedad más grave y se asocia con una mayor tasa de colectomía. La cicatrización de la mucosa se asocia con menores tasas de recaída y menos necesidad de tratamiento quirúrgico ${ }^{49}$.

Se ha demostrado que la actividad histológica persiste a pesar de los hallazgos endoscópicos normales. Algunas características histológicas, como plasmocitosis y linfocitosis basal e infiltrados inflamatorios crónicos, se han descrito en biopsias sin datos de actividad endoscópica, y se han relacionado con un mayor riesgo de recaída posterior (20-57\% $)^{49}$.

El número de nuevos casos ha aumentado significativamente en la última década en México, así como en otros países de América Latina ${ }^{7,17}$.
Las terapias tanto médicas como quirúrgicas empleadas en la CUCl se llevan a cabo con el objetivo de reducir la mortalidad, que ha disminuido del 2030\% hace $20-30$ años al 2-3\% en pacientes operados en la misma hospitalización. Las tasas de mortalidad permanecen por debajo del $1 \%$ si el tratamiento se realiza en centros especializados.

A pesar de las nuevas terapias médicas empleadas en la CUCl (esteroides, inmunosupresores y biológicos), la cirugía todavía desempeña un papel importante. Si bien la tasa de colectomías para la CUCI no aguda se ha reducido en un $50 \%$ en los últimos 5 años, la necesidad de tratamiento quirúrgico no ha presentado variaciones en la colitis aguda grave en los últimos 30-40 años ${ }^{8,9}$.

\section{Conclusiones}

En nuestro estudio, la actividad clínica persistente, la extensión pancolónica, el tratamiento con esteroides, la actividad endoscópica grave y la actividad histológica grave son predictores de colectomía. La gravedad medida por las escalas endoscópica de Mayo e histológica de Riley se asocia a un mayor riesgo de colectomía. La gravedad medida por la escala de Truelove y Witts no se asoció a colectomía. Por otra parte, no hubo asociación entre las características demográficas de nuestros pacientes y el riesgo de cirugía. Sin embargo, las características clínicas de la CUCl son útiles para identificar a los pacientes que pudieran presentar una evolución tórpida de la enfermedad con riesgo de requerir tratamiento quirúrgico.

\section{Conflicto de intereses}

Los autores declaran no tener ningún conflicto de intereses.

\section{Financiamiento}

La presente investigación no ha recibido ayudas específicas provenientes de agencias del sector público, del sector comercial ni de entidades sin ánimo de lucro.

\section{Responsabilidades éticas}

Protección de personas y animales. Los autores declaran que para esta investigación no se han realizado experimentos en seres humanos ni en animales. 
Confidencialidad de los datos. Los autores declaran que han seguido los protocolos de su centro de trabajo sobre la publicación de datos de pacientes.

Derecho a la privacidad y consentimiento informado. Los autores declaran que en este artículo no aparecen datos de pacientes.

\section{Bibliografía}

1. Podolsky DK. Inflammatory bowel disease. N Engl J Med. 2002;347:417-29

2. Sartor RB. Current concepts of the etiology and pathogenesis of ulcerative colitis and Crohn's disease. Gastroenterol Clin North Am. 1995;24:475-507.

3. Shanahan F. Inflammatory bowel disease: immunodiagnostics, immunotherapeutics and ecotherapeutics. Gastroenterology. 2001;120:622-35.

4. Lakatos $L$, Lakatos $P$. Is the incidence and prevalence of inflammatory bowel diseases increasing in Eastern Europe? Postgrad Med J. 2006:82:332-7.

5. Yang SK, Loftus EV, Sandborn WJ. Epidemiology of inflammatory bowel disease in Asia. Inflammatory Bowel Dis 2001;7:260-70.

6. Lakatos PL. Recent trends in the epidemiology of inflammatory bowel diseases: up or down? World J Gastroenterol 2006;12:6102-8.

7. Yamamoto-Furusho JK. Clinical epidemiology of ulcerative colitis in Mexico. A single hospital-based study in a 20 -year period (1987-2006). J Clin Gastroenterol. 2009;43:221-4.

8. Metcalf AM. Elective and emergent operative management of ulcerative colitis. Surg Clin North Am. 2007;87:633-41.

9. Ross H, Steele SR, Varma M, Dykes S, Cima R, Buie WD, et al. Practice parameters for the surgical treatment of ulcerative colitis. Dis Colon Rectum.2014;57:5-22.

10. Hano García OM, Andrade Gomes S, Villa Jiménez OM, González FL, Wood RL. Characterization of patients with ulcerative colitis treated at tertiary center. Revista Cubana de Medicina. 2016;55:46-58.

11. Loftus CG, Loftus Jr EV, Harmsen WS, Sandborn WJ. Update on the incidence and prevalence of Crohn's disease and ulcerative colitis in Olmsted County, Minnesota, 1940-2000. Inflamm Bowel Dis. 2007; 13:254-61.

12. Thukkani N, Sonnemberg A. Epidemiologic characteristics of patients with inflammatory bowel disease undergoing colonoscopy. Inflamm Bowel Dis. 2011;17:1333-7.

13. Molodecky NA, Panaccione R, Ghosh S, Barkema HW, Kaplan G, Environmental determinants of IBD. Clinical review. Inflamm Bowel Dis. 2011; 17:1992-8.

14. Yamamoto-Furusho JK, Rodríguez-Bores L, Granados J. HLA-DRB1 alleles are associated with the clinical course of disease and steroid dependence in Mexican patients with ulcerative colitis. Colorectal Dis. 2010;(12):1231-5

15. Yamamoto-Furusho JK, De-León-Rendón JL, de la Torre MG, Alvarez-León E, Vargas-Alarcón G. Genetic polymorphisms of interleukin 20 (IL-20) in patients with ulcerative colitis. Immunol Lett. 2013;149:50-3.

16. Yamamoto-Furusho JK, De-León-Rendón JL, Alvarez-León E, et al. Association of the interleukin 15 (IL-15) gene polymorphisms with the risk of developing ulcerative colitis in Mexican individuals. Mol Biol Rep. 2014;41:2171-6.

17. Bosques-Padilla FJ, Sandoval-García ER, Martínez-Vázquez MA Garza-González E, Maldonado-Garza HJ. Epidemiología y características clínicas de la colitis ulcerosa crónica idiopática en el noreste de México. Rev Gastroenterol Mex. 2011;76:34-8.

18. Cosnes J, Gower-Rousseau C, Seksik P, Cortot A. Epidemiology and natural history of inflammatory bowel diseases. Gastroenterology. 2011; 140:1785-94.

19. Prideaux L, Kamm MA, De Cruz PP, Chan FK, Ng SC. Inflammatory bowel disease in Asia: a systematic review. J Gastroenterol Hepatol. 2012;27:1266-80.

20. Calkins BM. A meta-analysis of the role of smoking in inflammatory bowe disease. Dig Dis Sci. 1989:34:1841-54.

21. Cosnes J. Tobacco and IBD: relevance in the understanding of disease mechanisms and clinical practice. Best Pract Res Clin Gastroenterol. 2004;18:481-96.

22. Velayos FS, Loftus EV, Jess T, Harmsen WS, Bida J, Zinsmeister AR, et al. Predictive and protective factors associated with colorectal cancer in ulcerative colitis: a case-control study. Gastroenterology. 2006; 130:1941-9.

23. Satsangi J, Silverberg MS, Vermeire S, Colombel JF. The Montreal classification of inflammatory bowel disease: controversies, consensus, and implications. Gut. 2006;55:749-53.
24. Molinié F, Gower-Rousseau C, Yzet T, Merle V, Grandbastien B, Marti R, et al. Opposite evolution in incidence of Crohn's disease and ulcerative colitis in Northern France (1988-1999). Gut. 2004;53:843-8.

25. Portela F, Magro F, Lago P, Cotter J, Cremers I, de Deus J, et al. Ulcerative colitis in a Southern European country: a national perspective. Inflamm Bowel Dis. 2010;16:822-9.

26. Ng SC, Tang W, Ching JY, Wong M, Chow CM, Hui AJ, et al. Incidence and phenotype of inflammatory bowel disease based on results from the Asia-pacific Crohn's and colitis epidemiology study. Gastroenterology. 2013;145:158-65.

27. Souza MH, Troncon LE, Rodrigues CM, Viana CF, Onofre PH, Monteiro RA, et al. Trends in the occurrence (1980-1999) and clinical features of Crohn's disease and ulcerative colitis in a university hospital in southeastern Brazil. Arq Gastroenterol. 2002;39:98-105.

28. Jiang XL, Cui HF. An analysis of 10,218 ulcerative colitis cases in China World J Gastroenterol. 2002;8:158-61.

29. Aghazadeh R, Zali MR, Bahari A, Amin K, Ghahghaie F, Firouzi F. Inflammatory bowel disease in Iran: a review of 457 cases. J Gastroenterol Hepatol. 2005;20:1691-5.

30. Silva BC, Lyra AC, Rocha R, Santana GO. Epidemiology, demographic characteristics and prognostic predictors of ulcerative colitis. World $\mathrm{J}$ Gastroenterol. 2014;20:9458-67.

31. Morales Polanco S, Waller González LA. Validación del índice de severidad endoscópico de colitis ulcerativa para la población mexicana. Endoscopia. 2016;28:2-8.

32. Travis SPL, Schnell D, Krzeski P, Abreu MT, Altman DG, Colombel JF, et al. Reliability and initial validation of the ulcerative colitis endoscopic index of severity. Gastroenterology. 2013;145:987-95.

33. Rogler G, Bernstein CN, Sood A, Goh KL, Yamamoto-Furusho JK, Abbas $Z$, et al. Role of biological therapy for inflammatory bowel disease in developing countries. Gut. 2012;61:706-12.

34. Pithadia AB, Jain S. Treatment of inflammatory bowel disease. Pharmacological Reports India. 2011;63:629-42.

35. Jiang L, Xia B, Li J, Ye M, Yan W, Deng C, et al. Retrospective survey of 452 patients with inflammatory bowel disease in Wuhan city, central China. Inflamm Bowel Dis. 2006;12:212-7.

36. Yamamoto-Furusho JK, Gutiérrez-Grobe Y, López-Gómez JG, Bosques-Padilla F, Rocha-Ramírez JL. Consenso mexicano para el diagnóstico y tratamiento de la colitis ulcerosa crónica idiopática. Rev Gastroenterol Mex. 2018:83:144-67.

37. Yamamoto-Furusho JK, Bosques-Padilla F, de-Paula J, Galiano MT, Ibáñez $P$, Juliao $F$, et al. Diagnosis and treatment of inflammatory bowel disease: First Latin American Consensus of the Pan American Crohn's and Colitis Organisation. Rev Gastroenterol Mex. 2017;82:46-84.

38. Stawowczyk E, Kawalec P. A systematic review of the cost-effectiveness of biologics for ulcerative colitis. Pharmacoeconomics. 2018;36:419-34.

39. Henriksen M, Jahnsen J, Lygren I, Sauar J, Kjellevold Ø, Schulz T, et al. Ulcerative colitis and clinical course: results of a 5 -year population based follow up study (the IBSEN study). Inflamm Bowel Dis. 2006;12:543-50.

40. Leijonmarck CE, Persson PG, Hellers G. Factors affecting colectomy rate in ulcerative colitis: an epidemiologic study. Gut. 1990;31:239-43.

41. Dias CC, Rodrigues PP, da Costa-Pereira A, Magro F. Clinical predictors of colectomy in patients with ulcerative colitis: systematic review and meta-analysis of cohort studies. J Crohns Colitis. 2015;9:156-63.

42. Shiga H, Takagi S, Inoue R, Kinouchi Y, Ohkubo T, Takahashi S, et al. What determines the later clinical course of patients who do not undergo colectomy at the first attack? A Japanese cohort study on ulcerative colitis. Digestion. 2010;81:104-12.

43. Samuel S, Ingle SB, Dhillon S, Yadav S, Harmsen WS, Zinsmeister AR, et al. Cumulative incidence and risk factors for hospitalization and surgery in a population-based cohort of ulcerative colitis. Inflamm Bowel Dis. 2013;19:1858-66.

44. Cosnes J. Smoking, physical activity, nutrition and lifestyle: environmental factors and their impact on IBD. Dig Dis. 2010;28:411-7.

45. Cañas-Ventura A, Márquez L, Ricart E, Domènech E, Gisbert JP, García-Sanchez V, et al. Risk of colectomy in patients with ulcerative colitis under thiopurine treatment. J Crohns Colitis. 2014:8:1287-93.

46. Solberg IC, Lygren I, Jahnsen J, Aadland E, Høie O, Cvancarova M, et al. Clinical course during the first 10 years of ulcerative colitis: results from a population-based inception cohort (IBSEN Study). Scand J Gastroenterol. 2009;44:431-40.

47. Aldhous MC, Drummond HE, Anderson N, Baneshi MR, Smith LA, Arnott ID, et al. Smoking habit and load influence age at diagnosis and disease extent in ulcerative colitis. Am J Gastroenterol. 2007:102:589-97.

48. Hoie O, Wolters FL, Riis L, Bernklev T, Aamodt G, Clofent J, et al. Low colectomy rates in ulcerative colitis in an unselected European cohort followed for 10 years. Gastroenterology. 2007;132:507-15.

49. Mohammed N, Subramanian V. Clinical relevance of endoscopic assessment of inflammation in ulcerative colitis: can endoscopic evaluation predict outcomes? World J Gastroenterol. 2016;22:9324-32. 